\title{
Towards a closure scheme for turbulent boundary layers using the attached eddy hypothesis
}

\author{
By A. E. PerRY, J. D. Li and I. MaruŠić \\ Department of Mechanical and Manufacturing Engineering, University of Melbourne, \\ Parkville, Victoria 3052, Australia
}

In this paper, an attempt is made to formulate a closure hypothesis for adverse pressure gradient turbulent boundary layers using the attached eddy hypothesis of Townsend and Perry \& Chong, which was developed originally for zero pressure gradient layers and parallel duct flows.

To the authors' knowledge, this work represents one of the few attempts to use coherent structure ideas in the formulation of a closure scheme. At present this closure scheme is primitive and many of the assumptions are of an arbituary nature but the analysis at least points out where the difficulties are and which areas need more work.

\section{Introduction}

In the past few decades, strong evidence has been presented, which suggests the existence of coherent structure in turbulent boundary layers (Kline et al. 1967). However, so far there are only a few papers which attempt to incorporate coherent structures in a closure model. By a closure model we mean a theoretical construction which gives a link between the mean velocity field and the turbulence field (in particular the Reynolds shear stress field). This enables the Reynolds momentum equations to be closed. There has been some work along this line which gives closure in certain regions. For instance, Walker et al. $(1987,1989)$ have constructed a model using a double roller eddy system in the viscous sublayer. The model proposed by Perry \& Chong (1982) is valid for flows beyond the viscous buffer zone. Here a brief review of the Perry \& Chong model is given.

Based on the Townsend attached eddy hypothesis (1976), the flow visualization experiments of Head \& Bandyopadhyay (1981) and Perry et al. (1981), Perry \& Chong (1982) have postulated that wall turbulence consists of a 'forest' of hairpin, horseshoe or ' $\Lambda$ ' vortices. It is generally believed that these vortices come from the viscous buffer zone. Helped by both vortex stretching and mutual induction, they lift themselves up from the wall and leave two long tails in the buffer zone. The two legs which form a ' $\Lambda$ ' shape, protrude into the turbulent wall region. They are inclined at approximately $45^{\circ}$ to the freestream velocity and lean in the downstream direction. The distance $\lambda_{0}$ between the two tails follows Kline's scaling (see Kline et al. 1967), i.e. $\lambda_{0} \approx 100 \mathrm{v} / U_{\tau}$ while the top of the ' $\Lambda$ '-shaped eddy is in a ' $\Omega$ ' shape (Hinze 1975). For simplicity, Perry \& Chong (1982) confined their attention to ' $\Lambda$ 'shaped eddies. Beginning with an isolated $\Lambda$-vortex, they showed that because of its image in the wall the vortex undergoes a stretching process in which the vortex height $h$ increases approximately uniformly with time and the distance $\lambda$ between the

Phil Trans. R. Soc. Lond. A (1991) 336, 67-79

Printed in Great Britain 
'legs' of the vortex at the wall decreases such that the product $\lambda h$ remains constant. They also showed that viscous diffusion ultimately dominates the stretching process and proposed that when the legs of the $\Lambda$-vortex eventually come together the vortex dies by vorticity cancellation. A random array of $\Lambda$-shaped vortices, all at different stages of stretching but with the same circulation, was called a 'hierarchy'. They found that in order to derive a logarithmic law for the mean velocity in the turbulent wall region, it is necessary to have different scales of hierarchies which are geometrically similar and with the same characteristic velocity scale $U_{\tau}$. Furthermore the PDF of these hierarchy scales must follow an inverse power law, the smallest hierarchy scaling with the viscous scaling being $c a .100 \mathrm{v} / U_{\tau}$ and the largest hierarchy with the boundary-layer thickness. Here the turbulent wall region is defined as $100 v / \delta_{H} U_{\tau}<z / \delta_{H}<0.15$, where $\nu$ is the kinematic viscosity, $\delta_{H}$ is the boundarylayer thickness, $U_{\tau}$ is the wall-shear velocity and $z$ is the local distance from the wall. The lower limit of this region is chosen for safety to ensure that the mean flow in this region follows the logarithmic law. How these hierarchies form is a mystery and vortex pairing has been suggested as a possible mechanism.

Perry et al. (1986) have extended this model to the whole turbulent boundary layer beyond the viscous region and found that the PDF of hierarchy or eddy length scales must be modified by a weighting function. This puts more weight on the large eddies so as to produce a mean-velocity-defect profile similar to that given by Hama (1954) or Coles (1956) beyond the logarithmic wall region. They also found that the attached eddies alone will not be sufficient to explain the energy dissipation. To account for this energy dissipation, they assumed that these attached eddies are surrounded by detached incoherent statistically isotropic and statistically irrotational assemblages of eddies which contribute to a classical Kolmogorov inertial subrange and dissipation range. These eddies are thought to be made up of the debris of broken up attached eddies. (The smallest eddies themselves carry with them some vorticity and therefore are rotational, but the space average (with the averaging box being the order of the attached eddy) of these eddies is irrotational.)

The attached eddy hypothesis has been found to be consistent with the dimensional analysis results as put forward by Perry \& Abell (1977) and Perry et al. (1986), in which the one-dimensional power spectral density $\phi_{11}\left(k_{1}\right)$ of the streamwise velocity fluctuation $u_{1}$ in the fully turbulent wall region has been found to have three identifiable ranges of the streamwise wavenumber $k_{1}$. These are an outer-flow scaling at low wavenumbers, an inter-flow scaling from low to moderate wavenumbers and a Kolmogorov scaling from moderate to high wavenumbers. A region of overlap between the outer-flow scaling and the inner-flow scaling is found to exist (overlap region I) which leads to a -1 power law for the power spectral density and at high wavenumbers a second overlap region exists (overlap region II) which gives the famous $-\frac{5}{3}$ power law. In connection with the attached eddy hypothesis, an identical analysis can be carried out for the spectrum $\phi_{22}\left(k_{1}\right)$ of the spanwise velocity fluctuation $u_{2}$ while for the spectrum $\phi_{33}\left(k_{1}\right)$ of the normal to the wall velocity fluctuation $u_{3}$, only overlap region II exists.

The above inverse power law spectrum and the well-known logarithmic mean velocity profile has been shown by Perry \& Chong (1982) to be consistent with having a range of geometrically similar hierarchies of the same velocity scale but whose length scale $\delta$ have an inverse power law PDF. Thus the mean velocity, most of the broadband turbulence intensity, the Reynolds shear stress, the turbulence energy spectra of different velocity components and the Reynolds shear stress spectrum

Phil. Trans. R. Soc. Lond. A (1991) 
from low to moderate wavenumbers can be calculated from the attached eddy model after assuming that the various hierarchies can be characterized by a random distribution of a fixed shape representative eddies with their length scales following the inverse PDF. Clearly this model can be used to find a connection between the mean flow field and the Reynolds shear stress and hence a closure scheme based on this model can be constructed.

Perry et al. $(1986,1987)$ and Perry \& Li $(1990)$ have presented some experimental data in turbulent pipe flows and in zero pressure gradient turbulent boundary layer flows over smooth and rough walls. Their results strongly support the attached eddy hypothesis and the associated scaling laws in these flows. Perry (1987) has reviewed the attached eddy model in zero pressure gradient turbulent boundary layer flows and a preliminary closure scheme based on this model has been suggested. Li (1989) and Perry et al. $(1988,1990)$ have extended the Perry \& Chong model (hereafter referred to as the $\mathrm{P} \& \mathrm{C}$ model) to adverse-pressure-gradient flows. In this paper, some details of this recent development are given and suggestions are made for further extensions to the theory.

\section{A modification to the $\mathrm{P} \& \mathrm{C}$ model}

In this section the detailed procedure of using the $\mathrm{P} \& \mathrm{C}$ model to relate the mean flow velocity and the Reynolds shear stress is given. It is shown that the original model (Perry et al. 1986) needs to be modified in order to obtained a Reynolds shear stress profile which is consistent with that of the momentum balance analysis. In modifying the $\mathrm{P} \& \mathrm{C}$ model, the shear stress profile of the zero pressure turbulent boundary layer at high Reynolds number has been used as a guide and at the same time physical explanations for the various modification have been given.

Perry et al. (1986) have shown that the mean-velocity-defect and Reynolds stress profiles are given by two convolution integral equations

$$
\begin{aligned}
\frac{\mathrm{d} U_{D}^{*}}{\mathrm{~d} \lambda_{E}} & =\int_{0}^{\lambda_{E}} M f(\lambda) \mathrm{e}^{-\lambda} q\left(\lambda-\lambda_{E}\right) \mathrm{d} \lambda, \\
\frac{\overline{u_{i} u_{j}}}{U_{\tau}^{2}} & =\int_{0}^{\lambda_{E}} M I_{i j}(\lambda) q\left(\lambda-\lambda_{E}\right) \mathrm{d} \lambda .
\end{aligned}
$$

Here $U_{D}^{*}=\left(U_{1}-U\right) / U_{\tau}, U_{1}$ is the freestream velocity, $U$ is the local mean velocity, $M$ is a universal constant to be determined, $f$ is the vorticity function of the representative eddy, $q$ is the weighting function, $\overline{u_{i} u_{j}}$ are the Reynolds stresses, $I_{i j}$ are the Townsend (1976) eddy-intensity functions of the representative eddy, $\lambda=$ $\ln (\delta / z), \lambda_{E}=\ln \left(\delta_{H} / z\right)$ and $\delta_{H}$ is the boundary layer thickness (which is assumed also to be the scale of the largest hierarchy).

Once the eddy shape is fixed, its vorticity function $f$ will be known and the eddy intensity functions $I_{i j}$ can be calculated from the Biot-Savart law. Hence (1) and (2) show that the mean flow and Reynolds shear stress are linked by the weighting function $q$. In this work Coles's $(1956,1962)$ mean velocity formulation is used, i.e. the law of the wall and law of the wake with the wake function as that given by Hinze (1959) (with a correction factor $\beta$ introduced, see Li \& Perry (1989)), thus

$$
\begin{gathered}
U / U_{\tau}=\kappa^{-1} \ln z U_{\tau} / \nu+A+(\Pi / \kappa)(1-\cos (\beta \pi \eta)) \\
\left(U_{1}-U\right) / U_{\tau}=-\kappa^{-1} \ln \eta+(\Pi / \kappa)(-\cos (\beta \pi)+\cos (\beta \pi \eta)) .
\end{gathered}
$$

Phil. Trans. R. Soc. Lond. A (1991) 

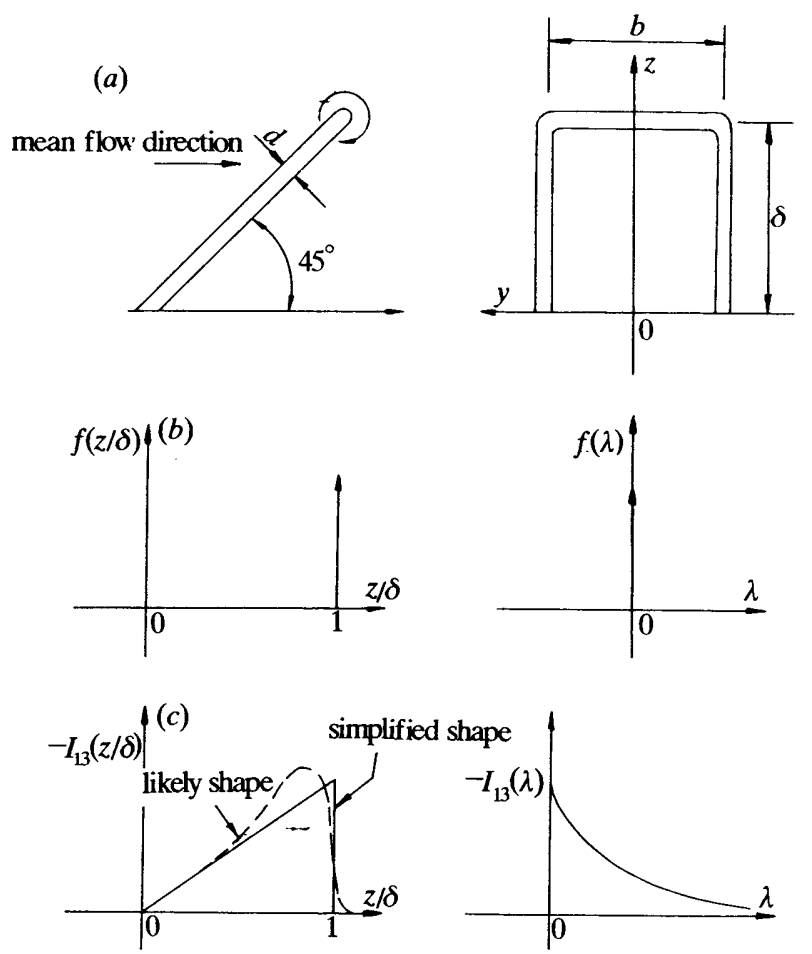

Figure 1. The assumed $\Pi$-shaped eddy. (a) Right, looking downstream left side view. (b) Vorticity function. As $d \rightarrow 0, f \rightarrow a$ Dirac delta function. (c) The simplified and a more realistic eddy intensity functions for Reynolds shear stress.

Here $\eta=z / \delta_{H}, \kappa$ is the Karman constant, $A$ is a universal constant, $\Pi$ is the wakestrength parameter, and the factor $\beta$ is a function of $\Pi$ and is introduced to ensure that mean-velocity gradient goes to zero as $\eta \rightarrow 1$. With the known vorticity function $f$ and (4), the weighting function $q$ can be found from (1) by a deconvolution process and the Reynolds shear stress can be calculated by a convolution calculation from (2) in conjunction with the eddy intensity function $I_{13}$.

For simplicity, it is assumed that each of the attached eddies in the turbulent wall flow can be represented by a characteristic ' $\Pi$-shaped' eddy as shown in figure 1 together with its vorticity function. The advantage of using ' $\Pi$-shaped' eddies in the analysis is that the vorticity function can be approximated as a Dirac delta function which greatly simplifies the mathematics. In assuming the representative eddy as that shown in figure 1, the long tails of the attached eddy have been neglected since they are mainly confined to the viscous zone (we have defined this as $0<z_{+}<100$ ). In this model the detailed structure of the viscous zone is neglected and replaced by a slip condition at the wall. Although this region contains the tails of the attached eddies, their contribution to the mean flow and turbulence quantities in the turbulent wall region is considered to be negligible. The eddy intensity function $I_{13}$ is assumed to be a linear function of $z / \delta$ as that shown in figure $1 c$ and this satisfies Townsend's (1976, p. 155) inviscid boundary condition near the wall. In fact it is this linear region near the wall which has the main influence on the convolution integral (2). The broken curve in figure $1 c$ is representative of results obtained using the Biot-Savart law for an isolated ' $\Pi$-shaped' eddy. Such departure from the linear 


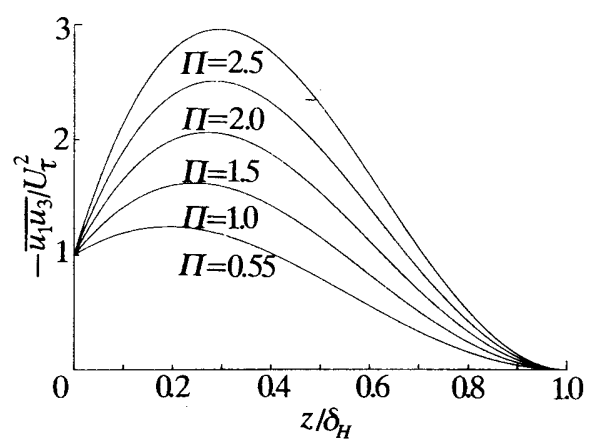

Figure 2. Reynolds shear stress profiles calculated from (5).

function does not alter significantly our conclusions concerning the shear stress distribution as discussed below.

Using the calculated weighting function $q,(2)$ and the linear function for $I_{13}$ as that shown in figure $1 c$, it can be shown that

$$
-\overline{u_{1} u_{3}} / U_{\tau}^{2}=(1-\eta)+\pi \Pi \eta \beta Y-\pi \Pi \beta \eta \mathrm{Si}(\pi \beta \eta)
$$

where

$$
\operatorname{Si}(x)=\int_{0}^{x} \frac{\sin (t)}{t} \mathrm{~d} t \quad \text { and } \quad Y=\operatorname{Si}(\beta \pi)
$$

In parallel duct flows, $\Pi \approx 0$, this reduces to the familiar linear Reynolds shear stress profile. Figure 2 shows the Reynolds-shear-stress profiles calculated from (5) with several $\Pi$ values. Since viscous contributions are restricted mainly to a layer of thickness $\delta_{1} \sim \nu / U_{\tau}$, then these contributions to the total shear stress will become negligible as $\delta_{1} / \delta_{H} \rightarrow 0$, which is equivalent to the Reynolds number (Kármán number) $\delta_{H} U_{\tau} / \nu \rightarrow \infty$. In the figure, $\Pi=0.55$ corresponds to infinite Reynolds number zero-pressure-gradient flows. Although the shear-stress profile corresponding to $\Pi=0.55$ satisfies the boundary conditions at both $\eta=0$ and $\eta=1$, the maximum value of the normalized Reynolds shear stress is larger than unity and occurs away from the wall. It will be seen in the momentum balance analysis carried out in $\S 3$ that such a profile cannot be correct.

Various representative eddy shapes have been tried to obtain the corresponding weighting functions (see Perry et al. 1986) and various eddy-intensity functions $I_{13}$ which satisfy the Townsend (1976) inviscid boundary condition have been used. So far no case has been found which will give a maximum normalized Reynolds shear stress at the wall for $\Pi=0.55$. The authors suspect that such an eddy shape does not exist and the weighting function $q$ needs to be modified.

In the P\&C model, it has been assumed that all the velocity scales are the same for different hierarchies. This may be true for duct or pipe flows, because in these flows the eddies which roll up from the buffer zone at the upstream station have the same wall-shear velocity as those which roll up from the downstream stations. In a turbulent boundary layer, this is no longer true, especially in flows with adverse pressure gradient which have the wall-shear velocity varying with streamwise distance. It is also a well-known fact that in turbulent boundary layer flows the Reynolds stress at a given station is influenced by the upstream history (Tani 1969), 
and many workers believe that Coles's wake component is a reflection of the upstream history of the layer, i.e. a 'memory' effect. So far the P\&C model has not taken this into account.

If one considers the eddies of different hierarchies at a streamwise location $X_{0}$ in a turbulent boundary layer flow, a sudden change in wall surface (e.g. from rough to smooth) at $X_{0}$ will cause the wall-shear velocity $U_{\tau}$ to change rapidly near $X_{0}$. This change of wall-shear velocity influences the velocity scale of the small hierarchy which has just rolled up from the buffer zone. The large hierarchies which come from upstream will not be affected greatly by this change. Instead they probably carry with them the velocity scales that come from the upstream stations. This example shows that different velocity scales should be introduced into different hierarchies for turbulent boundary layer flows.

Dimensional analysis suggests that the mean vorticity $\xi_{H}(z / \delta)$ from one hierarchy of the attached eddies can be expressed as

$$
\xi_{H}(z / \delta)=\left(U_{\tau i} / \delta\right) f(z / \delta)
$$

where $U_{r i}$ is the velocity scale of the hierarchy scale $\delta$. The mean vorticity from all hierarchies after being normalized with the local wall shear velocity $U_{\tau}$ is

$$
\frac{\mathrm{d} U / U_{\tau}}{\mathrm{d} z}=\int_{\delta_{1}}^{\delta_{H}} T\left(\delta / \delta_{H}\right) f(z / \delta) \frac{P_{H}(\delta)}{\delta} \mathrm{d} \delta,
$$

where $\delta_{1}$ is the length scale of the smallest hierarchy, $P_{H}(\delta)$ is the PDF of hierarchy length scales and $T=U_{\tau i} / U_{r}$. The function $T$ accounts for the characteristic velocity scale variation between the hierarchy scales and is assumed to be a function of $\delta / \delta_{H}$, i.e. it depends only on hierarchy scale. Following Perry et al. (1986), the PDF of eddy scales is still assumed to be

$$
P_{H}=(M / \delta) \omega\left(\delta / \delta_{H}\right)
$$

Here $\omega$ is the weighting function which represents only the departure of the PDF of hierarchy length scale from an inverse power law. When $\omega$ is unity $P_{H}$ is an inverse power law PDF which yields a logarithmic mean velocity profile. Thus for $\delta / \delta_{H} \rightarrow 0$, $\omega \rightarrow 1$. However for many cases, for $\delta / \delta_{H}$ larger than 0.10 (say) $\omega$ departs from unity and this represents eddy-population densities which depart from an inverse power law PDF.

Substituting (8) into (7) gives,

$$
\frac{\mathrm{d} U / U_{r}}{\mathrm{~d} z}=\int_{\delta_{1}}^{\delta_{H}} f\left(\frac{z}{\delta}\right) \frac{M}{\delta^{2}} T\left(\frac{\delta}{\delta_{H}}\right) \omega\left(\frac{\delta}{\delta_{H}}\right) \mathrm{d} \delta .
$$

It can be seen that effectively the original weighting function $q$ in (1) has been separated into $T\left(\delta / \delta_{H}\right)$ which represents the velocity-scale variation between different hierarchies and $\omega$ which represents the eddy-density departure from the inverse power law PDF.

Using a similar argument, it is simple to show that the Reynolds stresses are given by

$$
\frac{\overline{u_{i} u_{j}}}{U_{\tau}^{2}}=\int_{\delta_{1}}^{\delta_{H}} I_{i j}\left(\frac{z}{\delta}\right) \frac{M}{\delta} T^{2}\left(\frac{\delta}{\delta_{H}}\right) \omega\left(\frac{\delta}{\delta_{H}}\right) \mathrm{d} \delta
$$

and it should be noted that a $T^{2}$ is involved.

Phil. Trans. R. Soc. Lond. A (1991) 
Although the above modification to the $\mathrm{P} \& \mathrm{C}$ model can be justified physically, a close look at (9) and (10) shows that it has not solved the problem, i.e. the maximum normalized Reynolds shear stress-calculated from the model still occurs away from the wall for high Reynolds number zero-pressure-gradient flows. The reason for this is that higher velocity scales have been used for large hierarchies. Because of this, the maximum normalized Reynolds shear stress calculated from (9) and (10) is actually higher than that from (1) and (2).

To overcome this difficulty, another weighting function has been introduced and the weighting function will reduce the contribution to the total Reynolds stresses from larger hierarchies. One possible justification for this is that such a function might account for a possible change in the representative eddy shape with hierarchy scale. Some support for this will be discussed later. Also to keep the problem tractable we will consider a scenario where the eddies change their shape in such a way so as to alter the turbulence intensity and the Reynolds shear-stress profiles while preserving the velocity-defect profile. Hence the mean flow equation (8) is retained and the Reynolds stresses can be expressed as (repeated subscripts do not mean summation)

$$
\frac{\overline{u_{i} u_{j}}}{U_{\tau}^{2}}=\int_{\delta_{1}}^{\delta_{H}} I_{i j}\left(\frac{z}{\delta}\right) \frac{M}{\delta} S_{i j}\left(\frac{\delta}{\delta_{H}}\right) T^{2}\left(\frac{\delta}{\delta_{H}}\right) \omega\left(\frac{\delta}{\delta_{H}}\right) \mathrm{d} \delta,
$$

where $S_{i j}\left(\delta / \delta_{H}\right)$ is required as a factor which modifies $I_{i j}$ and for simplicity has been assumed to be a function of $\delta / \delta_{H}$ only. It should be pointed out that the Reynolds stresses are assumed to come entirely from the attached eddies and that contributions from the detached Kolmogorov inertial subrange eddies are small. This is probably true for the Reynolds shear stress but perhaps not true for the normal stresses.

The closure equations of the turbulent boundary layer flows after changing to the logarithmic variables as that used in (1) and (2) are

$$
\begin{gathered}
\frac{\mathrm{d} U_{D}^{*}}{\mathrm{~d} \lambda_{E}}=\int_{0}^{\lambda_{E}} M f(\lambda) \mathrm{e}^{-\lambda} \omega\left(\lambda-\lambda_{E}\right) T\left(\lambda-\lambda_{E}\right) \mathrm{d} \lambda, \\
\frac{\overline{u_{1} u_{3}}}{U_{\tau}^{2}}=\int_{0}^{\lambda_{E}} M I_{13}(\lambda) S_{13}\left(\lambda-\lambda_{E}\right) T^{2}\left(\lambda-\lambda_{E}\right) \omega\left(\lambda-\lambda_{E}\right) \mathrm{d} \lambda .
\end{gathered}
$$

In (12) and (13) after assuming $f(\lambda)$ and $I_{13}(\lambda)$ for a fixed eddy shape, one needs to separate $\omega, T$ and $S_{13}$ to solve these closure equations. The difficulty in using (12) and (13) is that the mean-flow velocity and the turbulence quantities are no longer directly related. Since $S_{13}$ is involved in the Reynolds shear-stress calculation, some knowledge about the Reynolds shear-stress profiles will help to construct it, hence leading to a tractable way of solving (12) and (13).

\section{Shear-stress profiles in two-dimensional turbulent boundary layer flows}

Here, analytical expressions for the shear-stress profiles in zero- and arbitrarypressure-gradient flows will be given and they are derived using the mean-flowmomentum and continuity equations in conjunction with the similarity laws (3) and (4). For the mathematical details, see Li (1989). Many people have attempted such an analysis, among them Coles (1952), Tetervin \& Lin (1955), Townsend (1956), Coles (1957) and Rotta (1962), all with some simplifying assumptions which we have 
avoided here. Some of the results concerning the zero-pressure-gradient flows have been given in Li et al. (1986) and Li \& Perry (1989). Granville (1988) also derived the shear-stress profiles for smooth and rough walls in zero-pressure-gradient flows but the asymptotic behaviour of these zero-pressure-gradient-shear-stress profiles was not discussed.

It is assumed that the turbulent boundary layer can be divided into a viscous zone (which includes the sublayer and the buffer zone) and an outer flow region. In the outer flow region the velocity profile can be written as

$$
\left(U_{1}-U\right) / U_{\tau}=f(\eta, \Pi) .
$$

Using the boundary-layer approximation and assuming two-dimensional flow, the mean momentum and continuity equations for zero-pressure-gradient flows are

$$
\begin{gathered}
U \partial U / \partial x+W \partial U / \partial z=\rho^{-1} \partial \tau / \partial z \\
\partial U / \partial x+\partial W / \partial z=0
\end{gathered}
$$

where

$$
\tau / \rho=\nu \partial U / \partial z-\overline{u_{1} u_{3}} .
$$

In (15) the term $\partial\left(\overline{u_{1}^{2}}-\overline{u_{3}^{2}}\right) / \partial x$ has been neglected. Here $W$ is the local mean velocity component normal to the wall, $\overline{u_{1}^{2}}$ is the streamwise Reynolds stress and $\overline{u_{3}^{2}}$ is the normal Reynolds stress. In further work it might be possible to include normal shear stress since there âre successful scaling schemes for the attached eddy contribution and the contribution from the Kolmogorov inertial subrange.

The boundary conditions are

$$
\begin{gathered}
U=W=0, \quad \tau=\tau_{0} \quad \text { at } \quad z=0, \\
U=U_{1}, \quad \tau=0 \quad \text { at } \quad z=\delta_{H} .
\end{gathered}
$$

In this paper, we are mainly interested in the shear-stress profiles outside the viscous zone. After some algebra, Li (1989) and Li \& Perry (1989) have shown that the shear stress profile can be expressed as

where $B=\kappa \sigma^{2} C_{1}-\kappa \sigma C_{2}+C_{2}$,

$$
\tau / \tau_{0}=1+C / B
$$

$$
\begin{gathered}
C=\kappa \sigma^{2} \eta f-\kappa \sigma^{2} \int_{0}^{\eta} f \mathrm{~d} \eta+\sigma \eta f-\int_{0}^{\eta} f^{2} \mathrm{~d} \eta-\kappa \sigma f \int_{0}^{\eta} f \mathrm{~d} \eta+\kappa \sigma \int_{0}^{\eta} f^{2} \mathrm{~d} \eta, \\
C_{1}=\int_{0}^{1} f \mathrm{~d} \eta, \quad C_{2}=\int_{0}^{1} f^{2} \mathrm{~d} \eta \quad \text { and } \sigma=U_{1} / U_{\tau} .
\end{gathered}
$$

In (19), a viscous correction term has been neglected. In doing this, we are effectively assuming that the mean velocity logarithmic law is valid down to $\eta=0$.

As Rotta (1962) has pointed out, the shear-stress profile (19) is not universal although a universal velocity-defect profile is used in deriving it. Since skin-friction coefficient approaches zero (i.e. $\sigma \rightarrow \infty$ ) as Reynolds number $U_{\tau} \delta_{H} / \nu$ approaches infinity, the shear-stress expression (19) can be simplified for infinite Reynolds number to

$$
\frac{\tau}{\tau_{0}}=1+\left[f \eta-\int_{0}^{\eta} f \mathrm{~d} \eta\right] / C_{1},
$$

which is a universal function of $\eta$ and is shown in figure 3 . The gradient of the shearPhil. Trans. R. Soc. Lond. A (1991) 


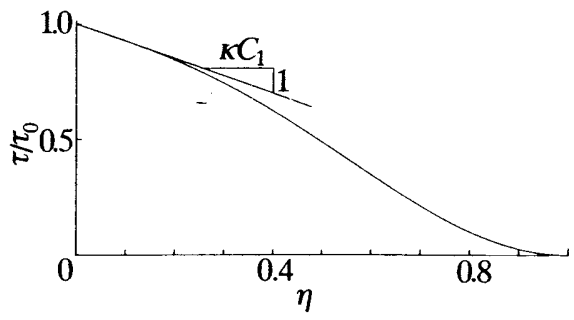

Figure 3. The total shear stress profile in zero pressure-gradient turbulent boundary layer flows when $R e \rightarrow \infty$ (equation (20)). Note the finite slope as $\eta \rightarrow 0$.

stress profile in the logarithmic region (which is defined as $\eta_{0} \leqslant \eta \leqslant 0.15$, it is also called the turbulent wall region) is not zero when the Reynolds number approaches infinity, but it is given by

$$
\partial\left(\tau / \tau_{0}\right) / \partial \eta=-1 / \kappa C_{1} .
$$

This behaviour of the shear stress profile has also been pointed out by Spalart (1988).

For adverse-pressure-gradient flows, we assume that the one parameter velocitydefect-profile family (14) is valid, $U_{1}=U_{1}(x), \Pi=\Pi(x)$ and the effect of the viscous zone is small. In the present work $\partial\left(\overline{u_{1}^{2}}-\overline{u_{3}^{2}}\right) / \partial x$ will be neglected but it should be included for flows approaching separation.

The momentum equation in arbitrary-pressure-gradient-boundary layers after applying the freestream condition is

$$
U \frac{\partial U}{\partial x}+W \frac{\partial U}{\partial z}=\frac{1}{\rho} \frac{\partial \tau}{\partial z}+U_{1} \frac{\partial U_{1}}{\partial x}
$$

$\mathrm{Li}(1989)$ has shown that the shear-stress profile for arbitrary-pressure-gradient turbulent boundary layer flows can be expressed as

where

$$
\frac{\tau}{\tau_{0}}=1+A_{1} \frac{\mathrm{d} \delta_{H}}{\mathrm{~d} x}+A_{2} \frac{\delta_{H} \mathrm{~d} \sigma}{\sigma \mathrm{d} x}+A_{3} \delta_{H} \frac{\mathrm{d} \Pi}{\mathrm{d} x}+A_{4} \frac{\delta_{H} \mathrm{~d} U_{1}}{U_{1} \mathrm{~d} x},
$$

$$
\begin{gathered}
A_{1}=\sigma \eta f-\sigma \int_{0}^{\eta} f \mathrm{~d} \eta-f \int_{0}^{\eta} f \mathrm{~d} \eta+\int_{0}^{\eta} f^{2} \mathrm{~d} \eta, \\
A_{2}=\sigma \int_{0}^{\eta} f \mathrm{~d} \eta-2 \int_{0}^{\eta} f^{2} \mathrm{~d} \eta+f \int_{0}^{\eta} f \mathrm{~d} \eta \\
A_{3}=-\left(\sigma \int_{0}^{\eta} \frac{\partial f}{\partial \Pi} \mathrm{d} \eta-\int_{0}^{\eta} \frac{\partial f^{2}}{\partial \Pi} \mathrm{d} \eta+f \int_{0}^{\eta} \frac{\partial f}{\partial \Pi} \mathrm{d} \eta\right), \\
A_{4}=-3 \sigma \int_{0}^{\eta} f \mathrm{~d} \eta+\sigma \eta f+2 \int_{0}^{\eta} f^{2} \mathrm{~d} \eta-f \int_{0}^{\eta} f \mathrm{~d} \eta .
\end{gathered}
$$

Equation (23) shows that the total shear stress is a function of $\delta_{H}, \Pi, \sigma$ and $U_{1}$ which are all functions of $x$.

\section{Attaining closure and a discussion about $S_{i j}$}

To separate $\omega$ and $T$ in (12) and (13), assumptions are needed. One way to separate $\omega$ and $T$ is to assume $\omega=1$, while $T$ can be obtained from (12) by a deconvolution method using a fast Fourier transform algorithm. $S_{13}$ can be obtained as follows. For 


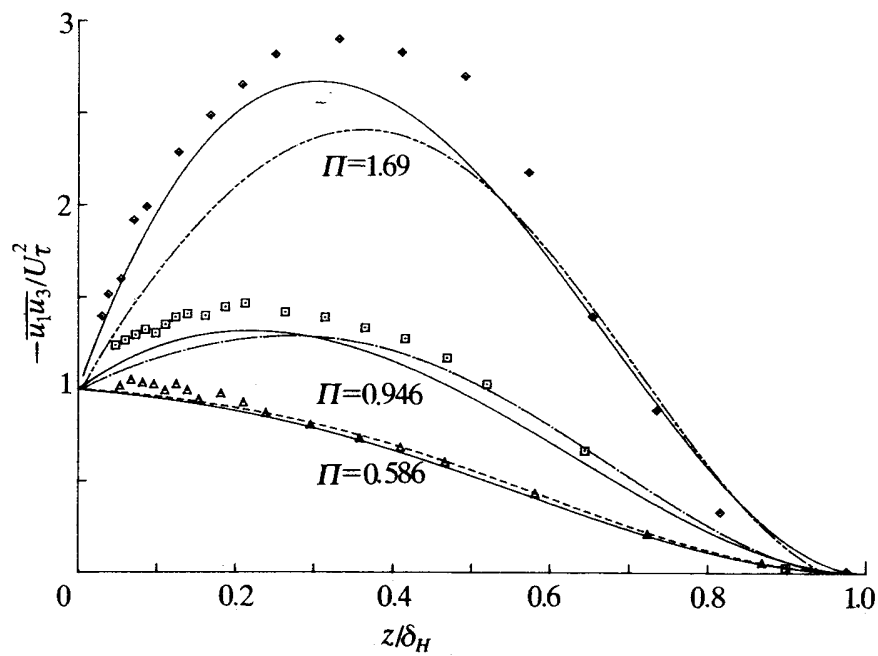

Figure 4. Reynolds shear stress profiles calculated from (13) (-_-, -._, - - . - ) and compared with the calculated results from (23) (-). Experimental data from East et al. (1979): $\triangle$, flow no. 3 ; 口, flow no. 4 ; $\diamond$, flow no. 5 .

convenience it has been assumed that $S_{13}$ is a universal function, i.e. it does not depend on the parameters $\Pi, \sigma$ and the various streamwise direction derivatives as mentioned in $\S 3$. The Reynolds shear-stress profile is a universal function in zeropressure-gradient turbulent boundary layer flow when Reynolds number approaches infinity. By substituting (20), $\omega$ and $T$ into (13), $S_{13}$ can be derived by using a deconvolution procedure (an analytical expression for $S_{13}$ can be derived under the above assumptions). The $S_{13}$ obtained from this is then put back into (13) and is used for calculating Reynolds shear stress for other flow cases. Hence (12) and (13) provide the necessary equations for closure.

Figure 4 shows the Reynolds shear-stress profiles calculated using (12) and (13) and these are compared with those calculated using (23). In calculating the profiles using (23), the mean-flow data of East et al. (1979) have been used and the direct viscous effect has been neglected. The data shown in the figure are from East et al. (1979). It can be seen that the curves calculated from both the model and the momentum balance agree well and they also agree well with the data up to $\Pi=1.69$. This is encouraging since it should be kept in mind that all constants were determined from the zero-pressure-gradient case of infinite Reynolds number.

Section 2 has shown that the inclusion of $S_{13}$ is necessary in order to give a reasonable result for the Reynolds shear-stress profiles. Perry (1987) conjectured that the single simple original weighting function $\omega\left(\delta / \delta_{H}\right)$ in (1) and (2) could include the effects of population density departure from the inverse power law, the velocityscale variation between hierarchies and a loss of geometrical similarity of the eddy shape. In the earlier work of Perry (1987), all of these effects were considered to be necessary but the conclusion here is that a single function is inadequate. In equations (12) and (13), we have assumed that $\omega$ represents the effect of population-density departure from the inverse power law and $T$ represents the velocity scale variation. $S_{13}$ has been assumed to be the effect from a loss of geometrical similarity of eddy shape from one hierarchy to another. The fact that $S_{13}$ or its equivalent does not appear in equation (12) means that we have restricted the way the eddy shape should 


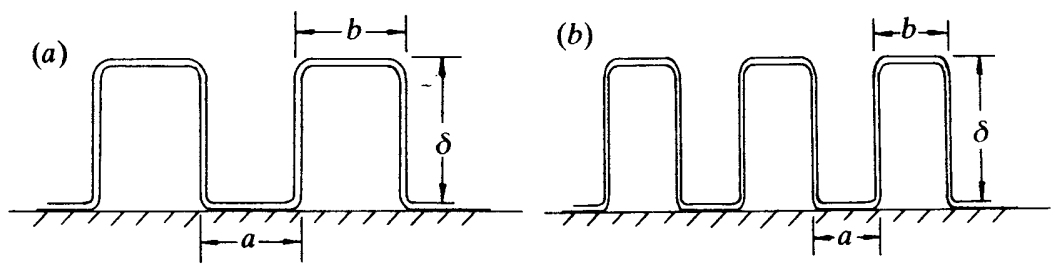

Figure 5. A suggestion for possible change in the eddy geometry from small hierarchy $(b)$ to large one $(a)$. In $(a)$ and $(b) a / b$ is the same but $b / \delta$ is different. These hierarchies have the same $\xi_{H}\left(z / \delta_{H}\right)$ but different $I_{23}(z / \delta)$.

be changed, i.e. we are effectively assuming that from one hierarchy to another the eddy structures are changing in such a way that this change will affect their contribution to Reynolds stresses but not to the mean vorticity.

Figure 5 illustrates one way in which this could occur. Here by an appropriate change in aspect ratio $b / \delta$, we keep the mean-vorticity distribution the same i.e. $\xi_{H}(z / \delta)$ is assumed unchanged but we vary $I_{13}$ hence the contribution to the Reynolds shear stress. There are many other geometrical variations which could occur and which need to be explored, e.g. a change in the fine scale features of the vortex core (e.g. the possible spiralling of vortex sheets within the core) and perhaps changes in the angle of inclination of the eddies to the wall and how these changes are related to the mean principle rate of strain axis. We have illustrated here the simplest possible geometrical change which upsets the often assumed one to one relationship between the mean flow field and the Reynolds shear stress field.

\section{Conclusions and discussion}

The attached eddy hypothesis is showing considerable promise in leading to a useful formulation for the prediction of turbulent boundary layer development with streamwise pressure gradients. It has been shown that the Perry \& Chong (1982) model needs to be modified to be applicable to flows other than in parallel ducts and pipes. By using the simple ' $\Pi$-shaped' eddy it is found that the velocity scale must vary with eddy-length scales and by doing this, some of the 'history' effects from upstream have been brought into the model. To obtain agreement with observed Reynolds shear-stress distributions, it is necessary to introduce yet another weighting function to reflect the variations in the contribution to the Reynolds stresses from different hierarchies. The physical meaning of this weighting function has been attributed to the loss of geometric similarity in the attached eddies as they grow from one hierarchy to another. To find a tractable way of separating various weighting functions, the eddy shape change has been restricted in the present paper such that only the contribution to the Reynolds stresses has been affected but not the meanflow velocity. Because of this restriction, the mean-flow-velocity-defect profile and the Reynolds stresses are no longer connected by the same weighting function and some knowledge about the shear-stress profile is necessary in order to separate the various weighting functions. The shear-stress profiles in zero- and adverse-pressuregradient flows have been derived by using Coles's $(1956,1962)$ law of the wall and law of the wake in connection with the boundary-layer equations. The shear-stress profile in zero-pressure-gradient flows approaches a universal one only when the Reynolds number approaches infinity. A closure is formed by using this profile along with the assumption that the weighting function $S_{13}$ is independent of Reynolds number and

Phil. Trans. R. Soc. Lond. A (1991) 
wake strength parameter $\Pi$. These closure equations have been used to calculate the Reynolds shear-stress profiles and these profiles are compared with the experimental data of East et al. (1979) as well as the shear-stress profiles calculated from the derived analytical expressions. Favourable agreements have been found in adversepressure-gradient flows up to $\Pi \approx 2$. Although it is impossible to know exactly how the characteristic eddies change their shapes from one hierarchy to another, some possible changes the eddies might undergo have been suggested by using intuitive arguments. It is hoped that a simple and universally valid formulation for the shape function $S_{i j}$ can be found so that an efficient closure scheme can be constructed.

The formulation given by (12) and (13) is consistent with the ideas expressed by Townsend (1961) that turbulence must be modelled with an account made for the fact that physical processes occurring at a point are related to processes remote from that point. In other words, the flow cannot be analysed on a point by point basis such as is implicit in those methods which use transport coefficients or other types of coefficients related to the local properties of the flow. The flow should really be analysed as an integrated whole with regard for the distribution of quantities remote from the point of interest. The convolution integral equations given by (12) and (13) satisfy these requirements.

The authors thank the Australian Research Council for the financial support of this project.

\section{References}

Coles, D. 1952 The law of the wall in turbulent shear flow. In 50 Jahre Grenzschichtforschung (ed. H. Gortler \& W. Tollmien), pp. 153-163. Vieweg: Braunschweig.

Coles, D. 1956 The law of the wake in the turbulent boundary layer. J. Fluid Mech. 1, 191-226.

Coles, D. 1957 Remarks on the equilibrium turbulent boundary layer. J. aero. Sci. 24, 495-506.

Coles, D. 1962 The turbulent boundary layer in a compressible fluid. USAF The Rand Cooperation, Rep. R-403-PR, Appendix A.

Granville, P. S. 1988 Eddy viscosities and mixing length for turbulent boundary layers on flat plates, smooth or rough. J. Ship Res. 32, 229-237.

Hama, F. R. 1954 Boundary layer characteristics for smooth and rough surfaces. Trans. Soc. nav. Arch. mar. Engrs 62, 333-358.

Head, M. R. \& Bandyopadhyay, P. R. 1981 New aspects of turbulent boundary-layer structure. J. Fluid Mech. 107, 297-338.

Hinze, J. O. 1975 Turbulence. New York: McGraw-Hill.

Kline, S. J., Reynolds, W. C., Schraub, F. A. \& Rundstadler, P. W. 1967 The structure of turbulent boundary layers. J. Fluid Mech. 30, 741-773.

Li, J. D. 1989 The turbulence structure of wall shear flow. Ph.D. thesis, University of Melbourne, Australia.

Li, J. D., Henbest, S. M. \& Perry, A. E. 1986 The difficulties in the measurements of Reynolds stresses in smooth- and in rough-wall turbulent boundary layers. Proc. 9th Australasian Fluid Mech. Con., Auckland, New Zealand.

Li, J. D. \& Perry, A. E. 1989 Shear stress profiles in zero-pressure-gradient turbulent boundary layers. Proc. 10th Australasian Fluid Mech. Conf., Melbourne, Australia.

Perry, A. E. 1987 Turbulence modelling using coherent structures in wakes, plane mixing layers and wall turbulence. Perspective in turbulence studies (ed. H. U. Meier \& P. Bradshaw). Springer.

Perry, A. E. \& Abell, C. J. 1977 Asymptotic similarity of turbulence structures in smooth- and rough-walled pipes. J. Fluid Mech. 79, 785-799.

Perry, A. E. \& Chong, M. S. 1982 On the mechanism of wall turbulence. J. Fluid Mech. 119, $173-217$.

Phil. Trans. R. Soc. Lond. A (1991) 
Perry, A. E., Henbest, S. M. \& Chong, M. S. 1986 A theoretical and experimental study of wall turbulence. J. Fluid Mech. 165, 163-199.

Perry, A. E. \& Li, J. D. 1990 Experimental support for the attached-eddy hypothesis in zeropressure-gradient turbulent boundary layers. J. Fluid Mech. 218, 405-438.

Perry, A. E., Li, J. D. \& Marusic, I. 1988 Novel methods of modelling wall turbulence. AIAA-880219.

Perry, A. E., Lim, K. L. \& Henbest, S. M. 1987 An experimental study of the turbulence structure in smooth- and rough-wall boundary layers. J. Fluid Mech. 177, 437-466.

Perry, A. E., Lim, T. T. \& Teh, E. W. 1981 A visual study of turbulent spots. J. Fluid Mech. 104, 387-405.

Perry, A. E., Li, J. D., Henbest, S. M. \& Marušić, I. 1990 Attached eddy hypothesis in wall turbulence. Near wall turbulence (ed. S. J. Kline \& N. H. Afgan), pp. 715-735. New York: Hemisphere.

Rotta, J. C. 1962 Turbulent boundary layer in incompressible flows. Prog. aero. Sci. 2, 1-220.

Spalart, P. R. 1988 Direct simulation of a turbulent boundary layer up to $R_{\theta}=1410$. J. Fluid Mech. 187, 61-98.

Tani, I. 1969 Review of some experimental results on the response of a turbulent boundary layer to sudden perturbations. Proc. 1968 AFOSR-IFP Stanford Conference on Comput. of Turb. Boundary-Layers (ed. S. J. Kline, G. Sovran, M. V. Morkovin \& D. J. Cockrell) 1, 483-494.

Tetervin, N. \& Lin, C. C. 1955 A general integral form of the boundary-layer equation for incompressible flow with an application to the calculation of the separation point of turbulent boundary layer. NASA Rep., p. 1046.

Townsend, A. A. 1956 The structure of turbulent shear flow, 1st edn. Cambridge University Press.

Townsend, A. A. 1961 Equilibrium layers and wall turbulence. J. Fluid Mech. 11, 97-120.

Townsend, A. A. 1976 The structure of turbulent shear flow, 2nd edn. Cambridge University Press.

Walker, J. D. A., Ece, M. C. \& Werle, M. J. 1987 An embedded function approach for turbulent flow prediction. $A I A A-87-1464$.

Walker, J. D. A., Abbott, D. E., Scharnhorst, R. K. \& Weigand, G. G. 1989 Wall-layer model for the Velocity Profile in turbulent flows. AIAA 27, 140-149.

Phil. Trans. R. Soc. Lond. A (1991) 


\section{University Library}

\section{- M M I N E R VA A gateway to Melbourne's research publications}

Minerva Access is the Institutional Repository of The University of Melbourne

Author/s:

Perry, A. E.;Li, J. D.;Marusic, I.

Title:

Towards a closure scheme for turbulent boundary layers using the attached eddy hypothesis

Date:

1991

Citation:

Perry, A. E., Li, J. D. \& Marusic, I. (1991). Towards a closure scheme for turbulent boundary layers using the attached eddy hypothesis. Philosophical Transactions of the Royal Society A, 336(1640), 67-79.

Publication Status:

Published

Persistent Link:

http://hdl.handle.net/11343/34801 